08

\title{
Переход заряда в вертикальных структурах, образованных двумерными слоями
}

\author{
(C) С.Ю. Давыдов ${ }^{1}$, А.А. Лебедев ${ }^{1}$, П.В. Булат ${ }^{2}$ \\ ${ }^{1}$ Физико-технический институт им. А.Ф. Иофрфе РАН, Санкт-Петербург, Россия \\ ${ }^{2}$ Севастопольский государственный университет, Севастополь, Россия \\ E-mail: Sergei_Davydov@mail.ru
}

Поступило в Редакцию 1 июня 2021 г.

В окончательной редакции 1 июня 2021 г.

Принято к публикации 5 июля 2021 г.

Методом функций Грина определены электронные структуры сверхрешеток и капсулированных слоев. Предложена простая схема оценки межслойного перехода заряда. В качестве примера рассмотрены слои графена и графеноподобных соединений.

Ключевые слова: 2D-структура, сверхрешетка, капсулированный слой.

DOI: 10.21883/PJTF.2021.20.51607.18896

Следствием возникновения графеновой тематики явился интенсивный поиск новых двумерных (2D) соединений и гетероструктур на их основе [1-3]. Сегодня известны сотни теоретически возможных 2D-материалов [4-6] и различные схемы формирования сверхрешеток (SL), образованных 2D-слоями (далее 2DSL) [7-9]. B [10] была предложена простая схема описания электронного спектра 2DSL, позволяющая учесть межслойное взаимодействие. Здесь мы используем результаты работы [10] для определения перехода заряда между образующими 2DSL слоями.

Рассмотрим вертикальную структуру, построенную из чередующихся листов 1 и 2, которые в изолированном виде описываются функциями Грина $G_{1,2}\left(\kappa_{1,2} ; \omega\right)$, где $\omega$ - энергетическая переменная, $\boldsymbol{\kappa}_{1,2}$ - волновые векторы для движения электронов в плоскости $(x, y, p d)$ листа $(p=0,1,2, \ldots N ; d-$ расстояние между слоями в 2DSL). Включим теперь межслойное взаимодействие $V$. В режиме слабой межплоскостной связи в диагональном приближении (см. [10]) функции Грина $\tilde{G}_{1,2}\left(\boldsymbol{\kappa}_{1,2} k_{z} ; \omega\right)$ слоев 2DSL можно представить в виде

$$
\begin{aligned}
& \tilde{G}_{1,2}^{-1}\left(\boldsymbol{\kappa}_{1,2}, k_{z} ; \omega\right)=G_{1,2}^{-1}\left(\boldsymbol{\kappa}_{1,2} ; \omega\right) \\
& -4 \cos ^{2}\left(k_{z} d\right) \Lambda_{2,1}(\omega)+i 0^{+}
\end{aligned}
$$

где $\Lambda_{1,2}(\omega)=V^{2} \int_{-\infty}^{\infty} \rho_{1,2}\left(\omega^{\prime}\right)\left(\omega-\omega^{\prime}\right)^{-1} d \omega^{\prime}-$ функции сдвига зонных состояний слоя 2 (1) под воздействием слоя 1 (2) [10], $\rho_{1,2}(\omega)=\pi^{-1} N_{\boldsymbol{\kappa}_{1,2}}^{-1} \sum_{\boldsymbol{\kappa}_{1,2}} \operatorname{Im} G\left(\boldsymbol{\kappa}_{1,2}\right)$ - плотности состояний свободных слоев 1 и $2\left(N_{\boldsymbol{\kappa}_{1,2}}-\right.$ числа состояний $\left.\boldsymbol{\kappa}_{1,2}\right), k_{z}$ - волновой вектор для движения электрона в вертикальном направлении (вдоль SL). Поскольку здесь мы не стремимся к получению точных результатов, а лишь проводим оценки межслойного перехода заряда, усредним выражение (1) по $k_{z}$. Тогда вместо (1) получим

$$
\begin{gathered}
\left\langle\tilde{G}_{1,2}^{-1}\left(\boldsymbol{\kappa}_{1,2}, k_{z} ; \omega\right)\right\rangle_{k_{z}}=\bar{G}_{1,2}^{-1}\left(\boldsymbol{\kappa}_{1,2} ; \omega\right) \\
=G_{1,2}^{-1}\left(\boldsymbol{\kappa}_{1,2} ; \omega\right)-2 \Lambda_{2,1}(\omega)+i 0^{+}
\end{gathered}
$$

Отметим, что сделанная замена обоснована в режиме слабой межслойной связи, так как $z$-зону можно считать узкой (бездисперсионной) по сравнению с 2D-зоной.

В качестве слоев, составляющих 2DSL, рассмотрим графен $(G r)$ и графеноподобные соединения (GLC). В низкоэнергетическом пределе плотность состояний свободного GLC и функция сдвига $\Lambda_{\mathrm{GLC}}(\omega)$ равны соответственно

$$
\begin{gathered}
\rho_{\mathrm{GLC}}(\Omega)= \begin{cases}2|\Omega| / \xi^{2}, & \Delta \leqslant|\Omega| \leqslant R, \\
0, & |\Omega|<\Delta,|\Omega|>R,\end{cases} \\
\Lambda_{\mathrm{GLC}}(\Omega)=\frac{2 V^{2} \Omega}{\xi^{2}} \ln \left|\frac{\Omega^{2}-\Delta^{2}}{\Omega^{2}-R^{2}}\right|,
\end{gathered}
$$

где $\Omega=\omega-\varepsilon, \xi=\sqrt{2 \pi \sqrt{3}} t, R=\sqrt{\xi^{2}+\Delta^{2}}, t$ - энергия перехода электрона между ближайшими атомами в слое, $2 \Delta$ - ширина запрещенной зоны свободного $\mathrm{GLC}, \varepsilon-$ энергия центра запрещенной зоны [11]. Положив $\Delta=0$ и $\varepsilon=\varepsilon_{D}$, где $\varepsilon_{D}-$ точка Дирака, получаем плотность состояний свободного графена $\rho_{G r}(\omega)$. Будем считать, что плотность состояний решеточных слоев $\bar{\rho}_{\mathrm{GLC}}$ определяется выражением (3), в котором $\Omega$ нужно заменить на $\bar{\Omega}=\omega-\bar{\varepsilon}$, где $\bar{\varepsilon}=\varepsilon+2 \Lambda_{n . n . l .}(\omega)$ и $\Lambda_{n . n . l .}(\omega)$ - сдвиг электронных состояний, вызванный ближайшим соседним слоем. Воспользуемся аппроксимацией, часто применяемой в теории адсорбции $[12]$, и положим $\bar{\varepsilon}=\varepsilon+2 \Lambda_{n . n . l .}(\varepsilon)$. Числа заполнения атомов слоев при нулевой температуре равны $\bar{n}_{1,2}=2 \int_{-\infty}^{\mu} \bar{\rho}_{1,2}(\omega) d \omega$, где $\mu-$ химический потенциал, 
положение которого определяется из условия сохранения заряда $\bar{n}_{1}+\bar{n}_{2}=n_{1}+n_{2}$, где $n_{1,2}$ - числа заполнения слоев в отсутствие взаимодействия. Межслойный переход заряда равен $v_{1,2}=\bar{n}_{1,2}-n_{1,2}$, откуда получаем $v_{1}=-v_{2}$.

Начнем с решетки, образованной слоями $G r$ и GLC, и будем считать $G r$ и GLC недопированными, так что $n_{1}=n_{2}=1$. Если $\bar{\varepsilon}_{D}<\bar{\varepsilon}-\Delta$, электроны переходят со слоев GLC на слои $G r$. При этом $v_{G r}=2\left(\mu-\bar{\varepsilon}_{D}\right)^{2} / \xi_{G r}^{2}$ и $v_{\mathrm{GLC}}=-2(\mu-\bar{\varepsilon})^{2} / \xi_{\mathrm{GLC}}^{2}$, откуда

$$
\begin{gathered}
\mu=\frac{\bar{\varepsilon}_{D} \xi_{\mathrm{GLC}}+\bar{\varepsilon} \xi_{G r}}{\xi_{G r}+\xi_{\mathrm{GLC}}}, \\
\nu_{G r}=2 \frac{\left(\bar{\varepsilon}-\bar{\varepsilon}_{D}\right)^{2}}{\left(\xi_{G r}+\xi_{\mathrm{GLC}}\right)^{2}} .
\end{gathered}
$$

Если $\bar{\varepsilon}_{D}>\bar{\varepsilon}+\Delta$, электроны переходят со слоев $G r$ на слои GLC. Тогда $v_{G r}=-2\left(\mu-\bar{\varepsilon}_{D}\right)^{2} / \xi_{G r}^{2} \quad$ и $v_{\mathrm{GLC}}=2(\mu-\bar{\varepsilon})^{2} / \xi_{\mathrm{GLC}}^{2}$, откуда вновь получаем выражение (4) для $\mu$, а для $v_{G r}$ нужно заменить знак плюс на минус. В том случае, когда $\bar{\varepsilon}-\Delta<\bar{\varepsilon}_{D}<\bar{\varepsilon}+\Delta$, перехода заряда не происходит. При $\varepsilon=\varepsilon_{D}$ (что приближенно соответствует графену и гексагональному нитриду боpa [10]) межслойный переход заряда также отсутствует. Оценки значений $t_{G r}$ и $t_{\mathrm{GLC}}, \Delta$ для $2 \mathrm{D}$-соединений $\mathrm{A}_{4} \mathrm{~B}_{4}$ и $\mathrm{A}_{3} \mathrm{~B}_{5}$ приведены в [13], значения $-\varepsilon$ и $-\varepsilon_{D}$ относительно вакуума равны соответствующим работам выхода. Вследствие разнообразия параметров вывод о том, как именно учет межслойного взаимодействия влияет на переход заряда, можно сделать только для конкретных 2DSL.

Для 2DSL, состоящих из слоев GLC1 и GLC2, $v_{1,2}= \pm 2\left(\mu-\bar{\varepsilon}_{1,2}\right)^{2} / \xi_{1,2}^{2}$, так что

$$
\begin{aligned}
& \mu=\frac{\bar{\varepsilon}_{1} \xi_{2}+\bar{\varepsilon}_{2} \xi_{1}}{\xi_{1}+\xi_{2}}, \\
& \nu_{1}=2 \frac{\left(\bar{\varepsilon}_{1}-\bar{\varepsilon}_{2}\right)^{2}}{\left(\xi_{1}+\xi_{2}\right)^{2}} .
\end{aligned}
$$

Если $\bar{\varepsilon}_{1}-\Delta_{1}<\bar{\varepsilon}_{2}-\Delta_{2}$, электроны переходят со слоев GLC2 на слои GLC1, при $\bar{\varepsilon}_{1}+\Delta_{1}>\bar{\varepsilon}_{2}+\Delta_{2}$ имеет место обратный процесс. При $\varepsilon_{1}=\varepsilon_{2}$ (что приближенно справедливо для листов $\mathrm{AlN}$ и $\mathrm{GaN}[10])$ переход заряда отсутствует.

Оценим теперь переход заряда в плоских капсулированных структурах. Для этого рассмотрим $N$ вертикально расположенных произвольных 2D-слоев, заключенных между массивными верхней (top) и нижней (bottom) обкладками. Воспользовавшись результатами работы [14], для функций Грина слоев $g_{p}(\omega)$, где $p-$ номер слоя, получим

$$
\begin{aligned}
& g_{N}^{-1}(\omega)=\omega-\varepsilon_{N}\left(\boldsymbol{\kappa}_{N}\right)-\Lambda_{t o p}(\omega)-\Lambda_{N-1}(\omega)+i 0^{+}, \\
& \begin{array}{c}
g_{p}^{-1}(\omega)=\omega-\varepsilon_{p}\left(\boldsymbol{\kappa}_{p}\right)-\Lambda_{p+1}(\omega)-\Lambda_{p-1}(\omega)+i 0^{+},
\end{array} \\
& 1 \leqslant p \leqslant N-1 \text {, } \\
& g_{1}^{-1}(\omega)=\omega-\varepsilon_{1}\left(\boldsymbol{\kappa}_{1}\right)-\Lambda_{2}(\omega)-\Lambda_{b o t}(\omega)+i 0^{+},
\end{aligned}
$$

где $\Lambda_{t o p(b o t)}(\omega)$ - сдвиг электронных состояний, наведенный верхней (нижней) обкладкой. В качестве примера рассмотрим капсулированный слой графена, находящийся между верхней металлической обкладкой (контактной площадкой) и нижней обкладкой - подложкой, представляющей собой полупроводник (например, $\mathrm{Si}, \mathrm{SiC}$ ) или диэлектрик (например, $\mathrm{BN}, \mathrm{SiO}_{2}$ ). Для немагнитного переходного металла (ТМ) имеем

$$
\begin{gathered}
\rho_{\mathrm{TM}}(\omega)= \begin{cases}10 / W_{d}, & \left|\Omega_{m}\right| \leqslant W_{d} / 2, \\
0, & \left|\Omega_{m}\right|>W_{d} / 2,\end{cases} \\
\Lambda_{\mathrm{TM}}(\omega)=\left(\Gamma_{m} / \pi\right) \ln \left|\frac{\Omega_{m}+W_{d} / 2}{\Omega_{m}-W_{d} / 2}\right|,
\end{gathered}
$$

где $\Omega_{m}=\omega-\varepsilon_{m} ; \Gamma_{m}=10 \pi V_{m}^{2} / W_{d} ; \varepsilon_{m}-$ энергия центра $d$-зоны ТМ, ширина которой равна $W_{d} ; V_{m}$ - матричный элемент взаимодействия металл-графен (модель Фриделя) [15]. Для полупроводника (диэлектрика) имеем

$$
\begin{gathered}
\rho_{\mathrm{SC}}(\omega)= \begin{cases}\rho_{s}, & \left|\Omega_{s}\right| \geqslant E_{g} / 2, \\
0, & \left|\Omega_{s}\right|<E_{g} / 2,\end{cases} \\
\Lambda_{\mathrm{SC}}(\omega)=\left(\Gamma_{s} / \pi\right) \ln \left|\frac{\Omega_{s}-E_{g} / 2}{\Omega_{s}+E_{g} / 2}\right|,
\end{gathered}
$$

где $\Omega_{s}=\omega-\varepsilon_{s}, \Gamma_{s}=4 \pi V_{s}^{2} / W_{s} ; W_{s}$ - ширина зоны проводимости и валентной зоны; $\varepsilon_{s}$ - энергия центра запрещенной зоны шириной $E_{g} ; V_{s}$ - матричный элемент взаимодействия подложка-графен (модель Халдейна-Андерсона) [16]. Полагая $\bar{\varepsilon}_{D}=\varepsilon_{D}+\Lambda_{\mathrm{TM}}\left(\varepsilon_{D}\right)+\Lambda_{\mathrm{SC}}\left(\varepsilon_{D}\right)$, получим $\left|v_{G r}\right|=2\left(\mu-\bar{\varepsilon}_{D}\right)^{2} / \xi_{G r}^{2}$, где положение $\mu$ задается обкладками. При $\mu<\bar{\varepsilon}_{D}$ имеем $v_{G r}<0$, т.е. происходит отток электронов из графенового слоя; при $\mu>\bar{\varepsilon}_{D}$ имеем $v_{G r}>0$, т.е. происходит приток электронов в графеновый слой. Возможен также случай, когда $\Lambda_{\mathrm{TM}}\left(\varepsilon_{D}\right)+\Lambda_{\mathrm{SC}}\left(\varepsilon_{D}\right)=0$ и $\bar{\varepsilon}_{D}=\varepsilon_{D}$.

Итак, в работе предложена простая схема оценки перехода заряда, позволившая получить аналитические результаты. Режим слабого (ван-дер-ваальсова) взаимодействия рассматривался не только из соображений простоты, но и на том основании, что только в таком режиме $G r$ и GLC сохраняют свои оригинальные свойства.

\section{Финансирование работы}

А.А. Лебедев и В.П. Булат признательны финансовой поддержке Министерства науки и высшего образования РФ в ходе реализации проекта „Фундаментальные основы механики, систем контроля и управления беспилотных авиационных систем с формообразующими конструкциями, глубоко интегрированными с силовыми установками, и уникальными свойствами, не применяемыми сегодня в пилотируемой авиации“ (№ FEFM-2020-0001). 


\section{Конфликт интересов}

Авторы заявляют, что у них нет конфликта интересов.

\section{Список литературы}

[1] A.K. Geim, I.V. Grigorieva, Nature, 499, 419 (2013). DOI: $10.1038 /$ nature 12385

[2] И.В. Антонова, ФТП, 50 (1), 67 (2016). [I.V. Antonova, Semiconductors, 50 (1), 66 (2016). DOI: $10.1134 / \mathrm{S} 106378261601005 \mathrm{X}]$.

[3] K.S. Novoselov, A. Mishchenko, A. Carvalho, A.H. Castro Neto, Science, 353, 9439 (2016). DOI: $10.1126 /$ science.aac 9439

[4] S. Haastrup, M. Strange, M. Pandey, T. Deilmann, P.S. Schmidt, N.F. Hinsche, M.N. Gjerding, D. Torelli, P.M. Larsen, A.C. Riis-Jensen, J. Gath, K.W. Jacobsen, J.J. Mortensen, T. Olsen, K.S. Thygesen, 2D Mater., 5, 042002 (2018). DOI: 10.1088/2053-1583/aacfe1

[5] N. Briggs, S. Subramanian, Z. Lin, X. Li, X. Zhang, K. Zhang, K. Xiao, D. Geohegan, R. Wallace, L.-Q. Chen, M. Terrones, A. Ebrahimi, S. Das, J. Redwing, C. Hinkle, K. Momeni, A. van Duin, V. Crespi, S. Kar, J.A. Robinson, 2D Mater., 6, 022001 (2019). DOI: 10.1088/2053-1583/aaf836

[6] L. Vannucci, U. Petralanda, A. Rasmussen, T. Olsen, K.S. Thygesen, J. Appl. Phys., 128, 105101 (2020). DOI: $10.1063 / 5.0021237$

[7] C. Forsythe, X. Zhou, K. Watanabe, T. Taniguchi, A. Pasupathy, P. Moon, M. Koshino, P. Kim, C.R. Dean, Nature Nanotechnol., 13, 566 (2018). DOI: $10.1038 / \mathrm{s} 41565-018-0138-7$

[8] P. Xiong, B. Sun, N. Sakai, R. Ma, T. Sasaki, S. Wang, J. Zhang, G. Wang, Adv. Mater., 32, 1902654 (2019). DOI: 10.1002/adma.201902654

[9] S. Liu, K. Yang, W. Liu, E. Zhang, Z. Li, X. Zhang, Z. Liao, W. Zhang, J. Sun, Y. Yang, H. Gao, C. Huang, L. Ai, P.K.J. Wong, A.T.S. Wee, A.T. N'Diaye, S.A. Morton, X. Kou, J. Zou, Y. Xu, H. Wu, F. Xiu, Natl. Sci. Rev., 7, 745 (2020). DOI: $10.1093 / \mathrm{nsr} / \mathrm{nwz} 205$

[10] С.Ю. Давыдов, ФТП, в печати.

[11] С.Ю. Давыдов, ФТП, $51 \quad$ (2), $226 \quad$ (2017). DOI: 10.21883/FTP.2017.02.44110.8336 [S.Yu. Davydov, Semiconductors, 51 (2), 217 (2017). DOI: $10.1134 / \mathrm{S} 1063782617020051]$.

[12] С.Ю. Давыдов, Теория адсорбиии: метод модельных гамильтонианов (Изд-во СПбГЭТУ „ЛЭТИ“, СПб., 2013). twirpx.com/file/1596114/

[13] С.Ю. Давыдов, ФТТ, 58 (4), 779 (2016). [S.Yu. Davydov, Phys. Solid State, 58 (4), 804 (2016). DOI: $10.1134 / \mathrm{S} 1063783416040041]$.

[14] С.Ю. Давыдов, Письма в ЖТФ, 47 (13), 52 (2021). DOI: 10.21883/PJTF.2021.13.51124.18749

[15] С.Ю. Давыдов, ФТТ, $62 \quad$ (2), $326 \quad$ (2020). DOI: 10.21883 FTT.2020.02.48886.602 [S.Yu. Davydov, Phys. Solid State, 62 (2), 378 (2020). DOI: $10.1134 / \mathrm{S} 1063783420020080]$.

[16] С.Ю. Давыдов, ФТП, 47 (1), 97 (2013). [S.Yu. Davydov, Semiconductors, 47 (1), 95 (2013). DOI: $10.1134 / \mathrm{S} 1063782613010090]$. 